\title{
How age of acquisition influences brain architecture in bilinguals
}

Miao Wei ${ }^{\mathrm{a}}$, Anand A. Joshi ${ }^{\mathrm{b}}$, Mingxia Zhang ${ }^{\mathrm{c}}$, Leilei Mei ${ }^{\mathrm{d}}$, Franklin R. Manis ${ }^{\mathrm{a}}$, Qinghua He ${ }^{\mathrm{a}}$,

Rachel L. Beattie ${ }^{\mathrm{e}}$, Gui Xue ${ }^{\mathrm{c}}$, David W. Shattuck ${ }^{\mathrm{f}}$, Richard M. Leahy ${ }^{\mathrm{b}}$, Feng Xue ${ }^{\mathrm{a}}$, Suzanne M.

Houston $^{\mathrm{a}}$, Chuansheng Chen ${ }^{\mathrm{g}}$, Qi Dong ${ }^{\mathrm{c}}$, Zhong-Lin Lu ${ }^{\mathrm{e}, *}$

${ }^{a}$ Department of Psychology, University of Southern California, Los Angeles, CA 90089-1061, USA.

${ }^{\mathrm{b}}$ Signal and Image Processing Institute, University of Southern California, Los Angeles, CA 90089-2564, USA.

${ }^{\mathrm{c}}$ National Key Laboratory of Cognitive Neuroscience and Learning, Beijing Normal University, Beijing 100875, China.

${ }^{\mathrm{d}}$ Center for Studies of Psychological Application and School of Psychology, South China Normal University, Guangzhou 510631, China.

${ }^{\mathrm{e}}$ Center for Cognitive and Behavioral Brain Imaging and Department of Psychology, The Ohio State University, Columbus, Ohio 43210, USA.

${ }^{\mathrm{f}}$ Ahmanson-Lovelace Brain Mapping Center, Department of Neurology, David Geffen School of Medicine, University of California, Los Angeles, CA 90095-7334, USA.

${ }^{\mathrm{g}}$ Department of Psychology and Social Behavior, University of California Irvine, Irvine, CA 92697, USA.

* Corresponding author. Phone: +1 6142478252 
Email address:1u.535@osu.edu

Mailing address: Department of Psychology, The Ohio State University, 1835 Neil Avenue Columbus, Ohio 43210, USA. 


\begin{abstract}
In the present study, we explored how Age of Acquisition (AoA) of L2 affected brain structures in bilingual individuals. Thirty-six native English speakers who were bilingual were scanned with high resolution MRI. After MRI signal intensity inhomogeneity correction, we applied both voxel-based morphometry (VBM) and surface-based morphometry (SBM) approaches to the data. VBM analysis was performed using FSL's standard VBM processing pipeline. For the SBM analysis, we utilized a semi-automated sulci delineation procedure, registered the brains to an atlas, and extracted measures of twenty four pre-selected regions of interest. We addressed three questions: (1) Which areas are more susceptible to differences in AoA? (2) How do AoA, proficiency and current level of exposure work together in predicting structural differences in the brain? And (3) What is the direction of the effect of AoA on regional volumetric and surface measures? Both VBM and SBM results suggested that earlier second language exposure was associated with larger volumes in the right parietal cortex. Consistently, SBM showed that the cortical area of the right superior parietal lobule increased as AoA decreased. In contrast, in the right pars orbitalis of the inferior frontal gyrus, AoA, proficiency, and current level of exposure are equally important in accounting for the structural differences. We interpret our results in terms of current theory and research on the effects of L2 learning on brain structures and functions.
\end{abstract}

Keywords: Age of acquisition; bilingual; structural plasticity; MRI 


\section{Introduction}

In the field of second language acquisition (SLA), the age of SLA onset attracts much less attention than the level of proficiency when researchers try to explore cortical representations of languages in bilinguals. The term "Age of Acquisition (AoA)" has been widely used to denote the age at which a monolingual individual first started learning a new or second language (Kovelman, Baker, \& Petitto, 2008). The effect of AoA is still entangled with that of the level of proficiency on cerebral organization in bilinguals (Wattendorf \& Festman, 2008). It has been shown that AoA modulates functional neural activity in several aspects of language processing, for example: phonology (Frenck-Mestre, Anton, Roth, Vaid, \& Viallet, 2005), syntax (Mahendra, Plante, Magloire, Milman, \& Trouard, 2003), different aspects of grammar (Arturo E. Hernandez, Hofmann, \& Kotz, 2007; Waldron \& Hernandez, 2013; Wartenburger, et al., 2003; Weber-Fox \& Neville, 1996) and lexical access (Isel, Baumgaertner, Thrän, Meisel, \& Büchel, 2010; Mahendra, et al., 2003; D. Perani, et al., 2003).

Growing evidence indicates that AoA is associated with fMRI BOLD activations in bilingual brains. For instance, in a narration task, Bloch et al. found that later AoA was associated with greater individual variations in the local cerebral activation of different languages in Broca's and Wernicke's areas (Bloch, et al., 2009). Likewise, Kim and colleagues reported that in Wernicke's area, identical regions serve both the first language (L1) and the second language (L2) in early and late bilinguals, but early bilinguals share overlapping L1 and L2 regions while late bilinguals have spatially distinct but neighboring L1 and L2 regions in Broca's area (K. H. Kim, Relkin, Lee, \& Hirsch, 1997). Other functional neuroimaging studies on effects of AoA suggest that late bilingual exposure is linked to a broader recruitment of neural 
tissues in the left inferior frontal gyrus (IFG; Hernandez, 2007; Kim, 1997), and bilateral IFG (Wartenburger, et al., 2003). Taken together, these studies suggest that AoA of L2 may have important effects on the functional organization of the language system in bilingual brains.

Functional neuroimaging methods such as PET and fMRI are widely used to study neural mechanisms in different cognitive skills. Although the traditional view is that experience and expertise with specific skills are mediated by functional (rather than structural) plasticity in the brain (K. H. Kim, et al., 1997), structural brain changes as a result of extensive experience in acquiring certain skills have been widely reported. For example, increased bilateral posterior hippocampal grey matter volume has been associated with acquisition of spatial representation in the London taxi driver study (Woollett \& Maguire, 2011). Likewise, medical students showed increased grey matter volume in the posterior and lateral parietal cortex bilaterally during their extensive medical examination study period (B. Draganski, et al., 2006). Structural changes related to bilingualism and multilingualism have also been reported. For example, bilinguals tend to have increased grey matter volume/density in Heschl's gyrus (Ressel, et al., 2012), the left caudate (Zou, Ding, Abutalebi, Shu, \& Peng, 2012), and the left inferior parietal structures (Della Rosa, et al., 2013; Mechelli, et al., 2004). Abutalebi, et al. (2013) showed that the only grey matter volume difference between early multilinguals and monolinguals was found in the left putamen and related to their proficiency levels of L3. However, upon careful review of the paper, one cannot rule out that the grey matter volume difference is rather due to both proficiency level and AoA.

In terms of second language AoA, at the whole brain level, Mechelli and colleagues reported higher grey matter density in the left inferior parietal lobule (IPL) in both early and late bilinguals compared to monolinguals. The effect was more evident in early bilinguals. These 
findings demonstrate changes in brain structures associated with second language learning.

However, with regard to L2 AoA, the right hemisphere has not received the same attention as the left hemisphere language regions, and hence, it has been relatively neglected by neuroscientists working in this field. This is surprising since in the most influential neuroimaging study on AoA by Mechelli et al, a trend of greater grey matter density was observed in the right IPL in early bilinguals (Mechelli, et al., 2004). Further, in neurosurgery, the right hemisphere structural plasticity has been reported widely (Duffau, 2006, 2014). However, whether and how changes in brain structures accompany variation in AoA has not been sufficiently investigated. Hence, it is evident that the right hemispheric neuroplasticity related to L2 AoA needs further study and clarification. In general, as pointed out by Abutalebi, et al. (2013), there are two major neural differences in L1 and L2 processing that were observed in the literature: one is L2's enhancing and sharing regions with L1 and the other is the specific engagement of areas that are outside of "traditional" left hemisphere language cortex that are recruited by the use of L2.

In the present study, we analyzed high resolution structural MRI images in brain regions implicated in prior structural and fMRI studies on second language learning in an attempt to gain insights into the nature of bilingual language processing and the impact of AoA on bilingual brains. We first undertook an exploratory whole-brain VBM analysis and then using SBM, we specified a set of ROIs based on existing literature, and performed statistical tests on the ROIs. Volume measures of 24 ROIs were obtained using a semi-automated sulci delineation procedure that registered the brains to an atlas to define the ROIs for each individual subject. Because a relatively small number of ROIs were used in SBM (surface-based morphometry), we only had to perform multi-comparison correction for a relatively small number of comparisons, leading to improved statistical power (Poldrack, 2007), which gave us another opportunity to discover 
subtle volume differences that may not survive the VBM whole-brain multiple comparison correction.

We sought to answer three questions. First, which of these brain regions are structurally affected by AoA? The adaptive control hypothesis by Green and Abutalebi (2013) predicted adaptive changes in the neural circuits associated with cognitive control processes in a duallanguage context. The authors provided a schematic description of the neural structures that were involved in language control processes. The cortical and subcortical "control network" included the prefrontal cortex (PFC), pre-supplementary motor area (pre-SMA), IFG, anterior cingulate cortex (ACC), caudate, putamen, thalamus and the parietal cortex. The model allows for bilateral structures such as bilateral inferior frontal cortex and bilateral basal ganglia, and predicts that earlier exposure to a bilingual environment would introduce more neural adaptation changes in this circuit. We first applied a whole-brain VBM search and then conducted a ROI analysis incorporating the regions that could possibly show AoA related volume changes according to the adaptive control hypothesis: the left PFC, left pre-SMA, the left IFG that is typically involved in effortful semantic retrieval (Fiez, 1997), left ACC, left caudate, left putamen, left thalamus and the left parietal cortex that is important for attention control (Della Rosa, et al., 2013). To provide a more detailed analysis, we divided the IFG into three sub-regions [pars opercularis (IFGop), pars triangularis (IFGpt), and pars orbitalis (IFGor)], the posterior parietal cortex into three sub-regions [supramarginal gyrus (SMG), angular gyrus (AG), and superior parietal lobule (SPL)], leading to a total of 12 ROIs in the left hemisphere. The selection of the 12 ROIs are also consistent with other previous studies (Arturo E. Hernandez \& Li, 2007; Jeong, et al., 2007; Mechelli, et al., 2004). Functionally, it has been generally accepted that more extensive left hemisphere activations are associated with bilinguals than monolinguals especially in late 
bilinguals and low proficiency bilinguals. But more and more studies also demonstrated involvement of right hemisphere homologous structures in bilinguals (Badzakova-Trajkov, Kirk, \& Waldie, 2008; Dehaene, et al., 1997; Wang, et al., 2011). Among the 12 ROIs we chose above, their right homologues, for example, the right IFG (also noted in the adaptive control hypothesis) and the right parietal cortex were repeatedly reported to have increased activation for L2 processing (Musso, et al., 2003; D. Perani, et al., 2003; Sebastian, Laird, \& Kiran, 2011; Wartenburger, et al., 2003). In terms of AoA of L2, a recent meta-analytic review also found that bilinguals who acquired both languages by 6 years of age demonstrated bilateral hemispheric involvement for both languages, whereas those who acquired their second language after age 6 showed left hemisphere dominance for both languages (Hull \& Vaid, 2007). Therefore, we included the right homologous regions of these 12 ROIs in our analyses. Structurally, the trend of greater grey matter density was observable in the right IPL in early bilinguals (Mechelli, et al., 2004). However, no other significant effects have been found in either grey or white matter.

Our second question was: how do AoA, proficiency and current level of exposure work together to shape bilingual brains? Various factors, such as linguistic distance between L1 and L2, nonnative status of the learner, L2 learner's language environment, years of education in L2, motivation for learning L2, language learning aptitude, age of L2 acquisition, degree of L2 proficiency, and relative L1 and L2 exposure, may modulate brain representations of languages in bilinguals (Higby, Kim, \& Obler, 2013; Klein, et al., 2006; Vaid, 1983). Among these variables, other than AoA, two factors, the degree of L2 proficiency (Chee, Hon, Lee, \& Soon, 2001; Chee, Soon, Lee, \& Pallier, 2004; De Bleser, et al., 2003; Elston-Guttler, Paulmann, \& Kotz, 2005; Mechelli, et al., 2004; D. Perani, et al., 2003; D. Perani, et al., 1998; Vingerhoets, et al., 2003; Wartenburger, et al., 2003) and the current level of exposure (D. Perani, et al., 2003; 
Vingerhoets, et al., 2003), have often been implicated in their effects on the neural organization of language processing in bilinguals (Consonni, et al., 2013). How these factors are related to structural changes in bilingual brains is still under debate. The current general consensus seems to be that differences in AoA, level of proficiency, type of grammatical construction and the amount of daily exposure to a language are generally reflected in the degree of activation but not in the localization of activation in functional imaging (Daniela Perani \& Abutalebi, 2005). It has been suggested that AoA has more influence on the neural substrate of L2 grammar processing compared to proficiency (Abutalebi, 2008; Wartenburger, et al., 2003), but the pattern of brain activity for semantic judgments depends strongly on proficiency (Indefrey, 2006; Daniela Perani \& Abutalebi, 2005; Wartenburger, et al., 2003). In this study, we evaluated effects of AoA, proficiency and current level of L2 exposure on the structures of bilingual brains.

The third question concerns the direction of structural brain differences. Do cortical regions become larger or smaller as AoA increases? Increased grey matter density in the midtemporal area and left posterior intraparietal sulcus has been shown after 3 months of juggling training (Bogdan Draganski, et al., 2004). Other than grey matter density, experience-induced cortical volume and/or thickness changes have also been reported. For example, researchers found that after 3 months of language learning, the volume of the right hippocampus, and the cortical thickness of the left middle frontal gyrus (MFG), left IFG and left superior temporal gyrus (STG) increased (Mårtensson, et al., 2012). Others reported that the volume of the posterior hippocampus correlated positively with the amount of time spent as a taxi driver, but the volume of the anterior hippocampus correlated negatively with the time spent as a taxi driver (Maguire, et al., 2000). As far as "early learning" is concerned, musicians showed increased grey matter volume in the left IFGop and the volume was positively correlated with years of musical 
performance (Abdul-Kareem, Stancak, Parkes, \& Sluming, 2011). It has also been reported that bilinguals who learned both languages before age 7 have larger Heschl's gyri volume than monolinguals (Ressel, et al., 2012). These results show that experience could either increase or decrease cortical volume and/or thickness. Although Mechelli and colleagues reported that the grey matter density in the left IPL decreased when the AoA of second language increased (Mechelli, et al., 2004), it is not completely clear how cortical volume or density varies with AoA. Based on the observation that skill acquisition alters grey matter volume in task-related areas and that $\mathrm{fMRI}$ studies showed broader activations in late bilinguals, our hypothesis is that larger grey matter volume would be associated with earlier AoA. Whether similar patterns would occur in the white matter, total volumes, cortical thickness and cortical surface area of the same regions is an open question. 


\section{Materials and Methods}

\subsection{Participants}

Thirty-six healthy native English-speaking bilingual adults were recruited from the University of Southern California and University of California, Irvine. Within these participants fourteen early bilinguals (mean age 22.3 \pm 2.6 , range 19-28) were exposed to their non-native language before they were 6 years old, four intermediate bilinguals (mean age $20.5 \pm 1.3$, range 19-22) acquired their non-native language between age 6 and 12, and eighteen late bilinguals (mean age $21.8 \pm 2.9$, range 18-30) were not exposed to their non-native language until they were 12 years old. The age cut-offs for the three groups were based on previous research (CansecoGonzalez, et al., 2010; Frenck-Mestre, et al., 2005; Wattendorf \& Festman, 2008). Demographics of early and late bilinguals are as follows: 1) Early: $6 \mathrm{M}$ and $8 \mathrm{~F}$; 4 Asian and $10 \mathrm{Westerner,} \mathrm{and}$ 2) Late: $8 \mathrm{M}$ and $10 \mathrm{~F}$; 4 Asian and 14 Westerner. Both parents belonged to the same ethnic group. All participants were right-handed according to the Edinburgh Inventory (Oldfield, 1971), had normal or corrected-to-normal vision and no history of head injury, psychiatric or neurological disorders. Informed consent was obtained from all participants, and all procedures were approved by the IRB on both sites.

\subsection{Language history questionnaire}

To render our findings independent of the effect of the type of the second language, we included participants with various non-native languages. Their second languages comprised Spanish (15), French (7), Mandarin (3), Cantonese (2), German (2), Vietnamese (2), Hebrew (1), Japanese (1), Portuguese (1), Serbian (1), and Urdu (1). In the present study, we controlled the 
similarity of the participants' two languages by balancing the second language type in the early and late bilinguals. AoA, language proficiency and current level of exposure of the native (English) and second languages were evaluated using self-reported measures in a language history questionnaire. Participants self-rated their native and second language proficiency on a seven-point scale (1= "only knows a little", 7= "native-level proficiency") in four different domains: reading, writing, listening, and speaking. To obtain a composite measure of native and second language proficiency, we computed the total scores of reading, writing, listening and speaking of each language. For both L1 and L2, the current level of exposure is evaluated by a question in the questionnaire "what is the overall percentage of time that you speak this language now?"

Previous studies suggested that bilinguals are able to assess their language proficiency and report their language history in a way that is consistent with behavioral performance (Jia, Aaronson, \& Wu, 2002). Grogan, et al. (2012) and Mayberry, Chen, Witcher, and Klein (2011) used a similar language history questionnaire in their work. All participants reported higher proficiency in English as shown in Table 1.

See Table 1

\subsection{Image acquisition}

Image data was obtained on a 3T Siemens Magnetom Trio MRI scanner located in the Dana and David Dornisife Cognitive Neuroscience Imaging Center at the University of Southern California. We acquired 3D, T1-weighted images using the Magnetization Prepared Rapid Gradient Echo (MP-RAGE) sequence and a 12-channel head coil. The scan parameters were: TR $2530 \mathrm{~ms}$, TE $3.09 \mathrm{~ms}$, TI $800 \mathrm{~ms}$, flip angle $10 \mathrm{deg}$, field of view $256 \mathrm{~mm}$, slice thickness $1 \mathrm{~mm}$, voxel dimensions: 1.0x1.0x1.0 mm, acquisition time $10 \mathrm{~min} 48 \mathrm{sec}$. 


\subsection{VBM and SBM analyses}

Two well-established techniques were available to detect the correlation between structure differences and language variables among subjects. VBM is a widely used automated technique which aligns the cortex of a subject to an atlas and requires no manual input. SBM, on the other hand, uses sulcal/gyral anatomy information for co-registration which can also provide additional information compared to VBM, such as cortical thickness, area, and curvature. VBM and SBM could generate different information and for the purpose of maximizing the robustness of our results, we choose both methods and report convergent findings.

\subsubsection{VBM}

Structural MRI data were analyzed with the Oxford Centre for Functional MRI of the Brain (FMRIB) software Library voxel-based morphometry (FSL-VBM), a VBM style analysis tool (Good, et al., 2001). After MRI signal inhomogeneity correction using the N4 method (http: //www.slicer.org), brains were first extracted, segmented and aligned to the MNI 152 standard space (Andersson, Jenkinson, \& Smith, 2007). Second, the spatially normalized images were then averaged to create a study-specific template, to which the native grey matter images were registered again using both linear and nonlinear algorithms. Third, the modulated grey matter images (to correct for local expansion or contraction due to the non-linear component of the spatial transformation) were then smoothed with an isotropic Gaussian kernel with a sigma of 3 $\mathrm{mm}$. The final measure of this procedure was grey matter volume.

\subsubsection{SBM}

BrainSuite is a surface-based image analysis tool that has been developed for MRI image and surface visualization, manual tracing of brain sulci, extraction of the inner and outer surfaces 
of the cerebral cortex, and segmentation of grey and white matter structures based on the 26 sulci drawn. We chose this approach because many volumetric-based approaches do not align the cortical anatomy well (Pantazis, et al., 2010) and we were interested in the cortical thickness, area and volume in functional areas of the cortex, which are closely related to the folding pattern.

\section{(a) Data pre-processing}

Our data analysis pipeline consisted of three steps: pre-processing, segmentation and registration. During pre-processing, we performed skull stripping, manual editing of the brain masks using BrainSuite11 (Shattuck \& Leahy, 2002) and N4 inhomogeneity correction.

We first imported the raw MRI data and used the BrainSuite Brain Surface Extractor (BSE) to complete skull and scalp stripping (Shattuck \& Leahy, 2002). BSE applied a combination of anisotropic diffusion filtering, edge detection and mathematical morphological (Dogdas, Shattuck, \& Leahy, 2005; Shattuck \& Leahy, 2002) operators to remove non-brain tissues in the MRI image. The interactive BSE tool was used to find the suitable parameters for brain extraction: diffusion iterations 5 , diffusion contrast 25 , edge constant 0.75 , and erosion size 1. We obtained a set of three-dimensional brain masks that excluded most non-brain tissues while preserving the cerebellum. Shattuck and Leahy reported that BSE could on average retain $99 \%$ of the white matter and grey matter in the final extracted brain masks with only $1 \%$ of nonbrain tissues (Shattuck \& Leahy, 2002). To make these masks even more accurate, we performed additional manual editing with the mask editing tool in BrainSuite. These edited masks were then saved for later intensity inhomogeneity correction purposes.

Imperfections in scanner hardware and variations in brain anatomy introduce intensity inhomogeneity to MR images that could confound the segmentation procedure (Shattuck, 
Sandor-Leahy, Schaper, Rottenberg, \& Leahy, 2001). We performed inhomogeneity correction using the N4ITK MRI Bias Correction module from 3D Slicer. Corrections were performed on the raw brain images using masks generated from the previous manual editing step since better inhomogeneity correction results could be obtained when the bias estimation is limited to a meaningful region. Further, we employed two sets of N4 parameters to optimize individual bias correction. Because N4's bias correction is a preprocessing step for tissue classification with its quality reflected in the performance of the tissue classification routine (Shattuck, et al., 2001), we chose between these two sets of parameters (See Table 2) based on the quality of tissue classification results from the next step, the set of parameters that yielded better tissue classification results for a particular participant was chosen for that participant.

See Table 2

To avoid group differences caused by N4 parameter differences, we balanced the number of participants using different parameters in our sample.

\section{(b) Brain Tissue Segmentation}

Next, we applied the Partial Volume Classifier (PVC) module in BrainSuite to perform tissue classification on the intensity-corrected brain. The algorithm labels each voxel in the image as background voxel, cerebrospinal fluid, grey matter and white matter. The labels were used to determine the volumes of each type of tissue.

The cerebrum was identified and extracted by registering a multi-subject average brain (ICBM452) to the individual brains using Automated Image Registration (AIR; (Pantazis, et al., 2010; Woods, Grafton, Holmes, Cherry, \& Mazziotta, 1998). We performed manual editing on the cerebrum masks and topology correction to the cortical surfaces. A graph-based approach 
was used to remove topological defects (handles and holes) and ensure a tessellation with spherical topology. Finally, brain pial, middle and inner cortex surfaces were generated. The inner cortex was expanded to form an outer/pial boundary. Results were carefully examined after each step. We returned to the previous step to improve parameter settings if necessary.

\section{(c) Cortical Surface and Volume Registration}

After cortical surfaces were generated from the last step, we split the brain into two hemispheres. Each hemisphere was then parameterized into a unit square map and the position in the unit square gave the coordinate for each vertex (A. Joshi, et al., 2009). Cortical surfaces from different participants were aligned in the 2D space and aligned to a template later in the registration step. Because the cytoarchitectural and functional parcellation of the cortex was intimately related to the folding of the cortex (A. Joshi, Shattuck, Thompson, \& Leahy, 2007), we aligned cortical surfaces by aligning meaningful cortical features/sulci first. The curve labeling protocol (Pantazis, et al., 2010) was followed in using the Curve Protocol Tool (A. A. Joshi, et al., 2010; Shattuck, et al., 2009) that has been integrated into BrainSuite software package. A thorough description of the sulcal curves with instructions on how to trace them is available on the website (http://neuroimage.usc.edu/CurveProtocol.html). The Curve Protocol Tool was an interactive tool to semi-automatically identify surface sulci. The rater was trained on the curve protocol by two experienced neuroanatomists (C.R. and S.Y.C.). Ten training sessions were scheduled ( 2 hours per session) to learn cortical anatomy and the tracing protocol. A set of 26 sulci on each hemisphere were delineated and used as landmarks in the surface matching procedure. The algorithm used a graph theory approach to identify the path between the start and end points of each sulcus specified by users with the lowest cost, which is defined as a combination of local curvature features and the distance between vertices on the surface 
representation (Shattuck, et al., 2009). We traced the curves with 0.5 stickiness (stickiness is associated with curvature weighting and it is a parameter to ensure that the paths of curves follow the sulcal depth of valleys) on the midcortical surface, midway between the pial surface and the grey-white interface, and when it was necessary to cross gyri, we used zero stickiness. Midcortical surfaces were used because they provided better access to the depth of sulci than pial surfaces, allowing more stable tracing (Pantazis, et al., 2010).

Finally, SVREG (Surface constrained Volumetric Registration), a set of programs that registers and labels BrainSuite generated cortical surfaces and volumes (A.A Joshi, Shattuck, \& Leahy, 2012), was used to segment the cortical surfaces and generate labels for each subregion. The program took BrainSuite-generated surfaces and volumes, registered them with the ICBM template, and segmented them into subregions with labels (since this data was processed, SVREG has been integrated with and is a part of newly released BrainSuite14b). Each hemisphere was divided into 47 cortical and subcortical ROIs (Table 3). The labeled surfaces were displayed in BrainSuite and carefully examined to make sure that desirable results were generated.

To work with the language control framework proposed by Green and Abutalebi (2013), three additional ROIs that were not included in the original 47 ROIs were manually delineated on the current template and further registered with SVREG. They were ACC, pre-SMA and PFC. The detailed descriptions of demarcation are illuminated here. ACC's caudal boundary as established by the mammillary bodies (Desikan, et al., 2006), pre-SMA was defined as the area of the medial frontal cortex in the superior frontal gyrus lying dorsal to the cingulate sulcus, rostral to the vertical commissure anterior (VCA) line, and caudal to the virtual line passing through the genu of the corpus callosum (J.-H. Kim, et al., 2010), whereas the PFC located 
anteriorly from the first slice that contained brain tissue and the posterior landmark was determined by first locating the most anterior slice that contained the temporal stem (the white matter tract connecting the temporal and frontal lobes (Wible, Shenton, Hokama, \& et al., 1995).

See Table 3 for all 50 ROIs on each hemisphere.

\subsection{Statistical Analysis}

\subsubsection{VBM}

A whole brain multiple regression analysis for all 36 subjects was performed with the FSL-VBM GLM model. AoA was the regressor of interest while proficiency and exposure were included as nuisance variables. Voxel-wise grey matter volume for the whole brain GLM was applied using permutation-based non-parametric testing, correcting for multiple comparisons across space.

\subsubsection{SBM}

The total volume, grey matter volume, white matter volume, thickness, and cortical surface area of the ROIs were computed by BrainSuite when available and exported to SPSS (version 17) for further analysis. The volume measures of each ROI were adjusted for total intracranial volume to account for head size. The adjustment was performed following a linear regression method (Buckner, et al., 2004; Chee, Zheng, Goh, Park, \& Sutton, 2011).

Previous research suggests that AoA and L2 proficiency are associated with neurofunctional organization in bilingual brains and are negatively correlated (DeKeyser \& LarsonHall, 2005 ). To evaluate separate effects of AoA and proficiency on cortical organization, we applied statistical partialing techniques (Birdsong, 2005) with AoA, proficiency, and current exposure level of L2 as three factors. Hypothesis-driven analyses were carried out on the set of 
24 pre-selected regions of interest (ROI): PFC, pre-SMA, IFGop, IFGpt, IFGob, anterior ACC, caudate, putamen, thalamus, SMG, AG, and SPL in both hemispheres.

The analysis was performed in two steps. In step one, AoA was included as the only predictor in the regression (correlation) models for all outcome measures, including grey matter volume, white matter volume, total volumes, thickness, and cortical surface area of each ROI. We then performed multiple comparison corrections with a false discovery rate (FDR) of 0.05 using Matlab programs written by ourselves (Storey, 2002) ${ }^{1}$. In step two, to further examine the effect of AoA on brain structures when proficiency and exposure are controlled, we included all three variables (AoA, proficiency and current exposure level of L2) as predictors in the ROIbased multiple regression models for the outcome measures of ROIs that survived the FDR correction. For all three variables, no extremely high correlation was found $(r>0.8)$.

${ }^{1}$ A variety of methods are available for multi-comparison correction. The most commonly used method, the Bonferroni correction, tends to eliminate both false and true positives in neuroimaging datasets (Genovese, Lazar, \& Nichols, 2002). FDR is a more liberal, yet powerful method (Storey, 2002). A recent study on age of language learning also used FDR to correct for multiple comparisons (Klein, Mok, Chen, and Watkins (2013). 


\section{Results}

\subsection{Exploratory VBM analyses}

No foci survived the whole-brain permutation-based correction for multiple comparisons. However, as shown in Figure 1, using a threshold of $\mathrm{p}=0.01$, we were able to identify a right parietal lobe cluster $(\mathrm{MNI}$ coordinates: $\mathrm{x}, \mathrm{y}, \mathrm{z}=42,-52,44$; cluster size $=116$ voxels; $\mathrm{t}$ score $=3.65$ ) of increased brain volume when the value of AoA was smaller controlling for both proficiency and exposure. It was located in the right AG and extended to the right SPL.

\section{See Figure 1.}

Figure 1. Volume increase with earlier second language acquisition. Increased grey matter volume in the right parietal lobe thresholded at $p=0.01$ (uncorrected). (a) axial view (b) sagittal view (c) coronal view.

\subsection{Hypothesis-driven SBM analyses}

For each ROI, we first entered AoA as the only predictor of our outcome variables (grey matter volume, white matter volume, total volume, thickness and cortical surface area of the ROIs) in distinct regression models. Regions that survived FDR multiple comparison correction are as follows. For total volume (the total of grey and white matter volume), three out of 24 ROIs, the right IFGor, the right AG and the right RSPL, survived the multiple testing correction $\left(p_{\text {corrected }}<0.05\right)$. For grey matter volume, two ROIs, the right IFGor and right SPL survived the correction. For white matter volume, two ROIs, the right IFGor and right AG survived the correction. For thickness, none survived the correction. For area, two ROIs, the right IFGor and 
right SPL survived the correction. The multiple regression model of the left AG was statistically significant for total, grey and white matter volumes but not strong enough to survive the FDR correction. The trend is that the earlier a non-native language was learned, the larger the volume in left AG. We then used multiple linear regression models for the ROIs that survived FDR correction to evaluate the relationship between different measures and AoA, proficiency and current exposure level (see results in Table 4).

\section{See Table 4}

Since all three variables were entered in the prediction model, it is possible to evaluate independent contributions of each variable. Overall, none of the three variables made a significant independent contribution in the right IFGor. In the right AG and right SPL, AoA did provide a significant independent contribution to the regression model while proficiency and current L2 exposure level did not.

In the right IFGor, the overall regression model was statistically significant for the total volume [R square $=0.302, \mathrm{~F}(3,32)=4.610, p=0.009]$, grey matter [R square $=0.299, \mathrm{~F}(3,32)$ $=4.545, p=0.009]$, white matter volume $[\mathrm{R}$ square $=0.236, \mathrm{~F}(3,32)=3.292, p=0.033]$ and cortical area $[\mathrm{R}$ square $=0.308, \mathrm{~F}(3,32)=4.162, p=0.015]$. In other words, these measures of the right IFGor are significantly correlated with AoA, L2 proficiency and current level of L2 exposure. However, none of the three predictors made a statistically significant contribution.

In the right $\mathrm{AG}$, the overall regression was statistically significant for the total ROI volume $[\mathrm{R}$ square $=0.254, \mathrm{~F}(3,32)=3.639, p=0.023]$. Thus, the total volume of right AG could be predicted at levels significantly above chance from AoA, L2 proficiency and level of exposure combined (that is when AoA, proficiency and exposure were used as predictors, about 
$25.4 \%$ of the variance in right AG volume could be predicted). In addition, AoA as a predictor variable made a statistically significant contribution. AoA was significantly predictive of the right AG total volume when proficiency and exposure were statistically controlled: $\mathrm{t}(32)=-$ $2.654, \mathrm{~b}=-230.971, p=0.012$. The negative slope indicated that there was about a $230 \mathrm{~mm}^{3}$ decrease in right AG volume for each 1-year increase in the age of L2 acquisition, controlling for proficiency and exposure. For the predictor variable proficiency $(\mathrm{b}=-177.964, \mathrm{t}=-1.738, p=0.092)$ and level of exposure $(b=-202.164, t=-0.038, p=0.970)$, there were no significant contributions. So AoA was a stronger predictor of the total volume of the right AG than proficiency and exposure. About $16 \%$ of the variance in the total volume of the right AG was uniquely predicted from AoA (when proficiency and exposure were statistically controlled). In addition, we found proficiency accounted for $7 \%$ of the variance by itself (when AoA and current L 2 exposure level were controlled) and current L2 exposure level did not provide significant additional contribution. For white matter volume measures, in right AG, the overall model was statistically significant, with $\mathrm{R}$ square $=0.315, \mathrm{~F}(3,32)=4.900, p=0.006$. AoA made a significant contribution $(\mathrm{b}=-$ 93.862, $\mathrm{t}=-3.148, p=0.004)$ and explained about $21 \%$ of variance in the white matter volume of the right AG when proficiency and exposure were controlled. Proficiency accounted for an additional 5\% of the variance (when AoA and exposure level were controlled) and exposure level did not explain any additional variance.

In the right SPL, the overall regression was statistically significant for total ROI volume $[\mathrm{R}$ square $=0.333, \mathrm{~F}(3,32)=5.337, p=0.004]$. Hence, the total volume of the right SPL could be predicted at levels significantly above chance from AoA, L2 proficiency and level of exposure combined. In addition, AoA was significantly predictive of right SPL total volume when proficiency and exposure were statistically controlled: $\mathrm{t}(32)=-3.008, \mathrm{~b}=-178.670, p=0.005$. AoA 
was a stronger predictor than proficiency and exposure and contributed about $19 \%$ variance to the total volume. Proficiency accounted for $3 \%$ of the variance and exposure level only explained $0.6 \%$ unique variance. Similarly, the overall model of grey matter volume of the right SPL was reliably significant $[\mathrm{R}$ square $=0.352, \mathrm{~F}(3,32)=5.792, p=0.003]$. AoA was the only significant predictor $[\mathrm{t}(32)=-3.097, \mathrm{~b}=-120.590, p=0.004]$ and it contributed about $19 \%$ variance for grey matter volume. Proficiency accounted for $4 \%$ of the variance and exposure level only explained $0.7 \%$ unique variance. Moreover, for the cortical area measure, AoA was significantly predictive of the right SPL contributing an $18 \%$ of the variance while proficiency accounted for $0.7 \%$ of the variance and exposure level explained $0.5 \%$ unique variance.

To further elucidate their influence on volume measures, separate scatter plots of volumes vs AoA, proficiency and exposure are provided in Figure 2.

Figure 2 shows that, in the right IFGor (a), both AoA and exposure are significantly correlated with total, grey and white volume measures. Positive correlations were found between AoA and the volumes while negative correlations occurred between exposure and the volumes. In other words, when the age of non-native language acquisition increased, the volume measures of right IFGor increased and when the exposure to L2 increased, the volumes decreased $(p<0.01)$. For the two parietal regions, right AG and right SPL (b and c), out of three predictors, AoA played a crucial role in grey matter, white matter and total volumes with only one exception, that is, proficiency was positively associated with grey matter volume of the right SPL. Differently from the right IFGor of the frontal lobe, the two parietal areas showed negative correlations in terms of AoA and volumes measures, that is, when the age of L2 acquisition increased, the grey matter, white matter and total volumes were smaller. 
See Figure 2

Figure 2. Scatter plots of the association between the volumes of selected regions and AoA, proficiency, exposure. WM=white matter; GM=grey matter. From anterior to posterior regions: (a) The right pars orbitalis. (b) The right angular gyrus. (c) The right superior parietal lobule (Correlation coefficient significance: $p<0.05^{*}, p<0.01^{* *}$ ).

\section{Discussion}

Following the line of investigation of fMRI studies that focused on the AoA effect, we concentrated on structural variations. We were able to find significant and consistent effects using two different whole-brain analysis approaches (VBM and SBM). The results suggest that we had adequate statistical power to detect reliable effects. We observed that neuroanatomical structures differed as a function of the age of second language acquisition in bilingual brains. The analyses generated the following answers to our questions: (1) Where? Which brain structures are more susceptible to differences in AoA? Out of the 24 pre-specified cortical regions, we found that volumetric measures of the right AG and right SPL plus the cortical area of right SPL in the parietal lobe were reliably sensitive to the age of second language acquisition. Our VBM analysis was consistent with this SBM finding. (2) How? How do AoA, proficiency and current level of exposure work together to shape bilingual neural representation? The bilingual brain organization is more dependent upon AoA in the right AG and SPL. In the right IFGor, AoA, proficiency and current level of exposure were related to structural differences but none of them was significantly stronger than the other two factors. (3) What? What is the effect of AoA on structural measures of brain structures? The earlier a non-native language was learned, the larger the volumes/area of right AG and right SPL were. For the left AG, the trend of the 
effect was that the earlier a non-native language was learned, the larger the left AG volume was. We discuss these findings in the following paragraphs.

\subsection{Areas showing AoA effect}

In the parietal lobe SBM analysis, we found that the volume measures of the right AG and right SPL were predicted by AoA, proficiency and exposure combined, and AoA as a predictor variable made a statistically significant contribution. Our cortical area data from SBM also supported the AoA's effect in the right SPL. These findings are consistent with the VBM analysis which found negative correlations between grey matter volume and AoA in a cluster located at the right AG extended to the SPL. The multiple regression model of the left AG was statistically significant for total, grey and white matter volumes but not strong enough to survive the FDR correction. The angular gyrus is located in the posterior part of the inferior parietal lobule and is posterior to the supramarginal gyrus. The bilateral posterior parietal cortex was found to be involved in the conflict resolution network including the ACC, dorsolateral PFC, IFG, posterior parietal cortex, and anterior insula (Nee, Wager, \& Jonides, 2007). Similarly, the bilateral posterior SPL was found to be significantly activated in an attention shifting task (Le, Pardo, \& Hu, 1998). Gurd, et al. (2002) proposed that the parietal lobe was involved in switching between language tasks. Bilinguals experience switching between their two languages on a daily basis, so it is reasonable to anticipate the need in bilinguals to recruit the parietal lobe. AG's grey matter maturation peaks between 8.5 and 13 years of age (Seghier, 2013). So the maturation peak of AG arrives after early bilinguals started to learn a second language (before 6 years old) and this may give the brain an opportunity to recruit additional resources (e.g., right AG). In early bilinguals, increased GM volume, WM volume, total volume and surface area relative to later bilinguals could correspond to greater computational power in the parietal cortex leading to a 
more efficient language-switching system and thus more efficient processing of two languages for early compared to late learners.

There are at least two ways to interpret the fact that the volumes of these right parietal regions were more strongly associated with AoA than the left parietal regions. $\underline{\text { Yoon, Fahim, }}$

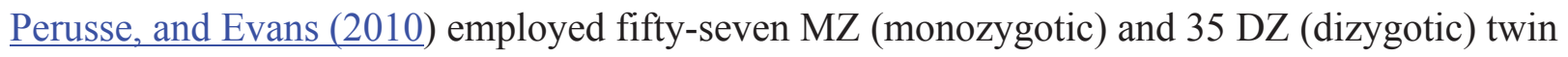
pairs and did MRI scans when they were 8 years old. It was found that genetic and environmental influences on individual human brain structural differences were lateralized, with the language-dominant left cerebral cortex under stronger genetic control than the right. Therefore, the language-related regions in the right hemisphere might be more vulnerable to the type of the environmental changes that might be reflected in AoA effects, as suggested by our results. Another way to interpret the recruitment of the right parietal regions is that neural plasticity in the parietal lobes of bilinguals largely depends on brain maturation. When brain resources are relatively limited during early bilingual learning, the right homologous language structures may be recruited, suggesting a compensatory role for right hemisphere regions while the left regions have to be used under any context. Some researchers have proposed that bilinguals had more overall right-hemisphere involvement for L2 and therefore weaker language lateralization compared to monolinguals (Dehaene, et al., 1997; Park, Badzakova-Trajkov, \& Waldie, 2012). Furthermore, as pointed out by Arturo E. Hernandez (2009) “it is very interesting that L1 effects appear as left lateralized whereas L2 effects appear as right lateralized". Existing evidence converge on the same point that being a bilingual, especially an early bilingual, may recruit more right hemisphere regions during language tasks and thus some right cortical regions may readily manifest the AoA effect. These explanations are admittedly post hoc in the case of our results, but merit further investigation. 
We found that the multiple regression model of the left AG was statistically significant for total, grey and white matter volumes but not strong enough to survive the FDR correction. The earlier a non-native language was learned, the larger the volume in left AG. From evolutionary perspective, we speculated that the reason of a generally weaker correlation of AoA and left AG volumes was because that the left hemisphere played a more important role in language and it may have to maintain its invariance structurally across various environmental conditions. However, this claim will require future research to support.

\subsection{How do AoA, proficiency and current level of L2 exposure work together to shape bilingual brains?}

Learning two languages simultaneously is a challenging task and thus may require taking full advantage of neural plasticity during childhood in early bilinguals (Peng \& Wang, 2011). However, the cerebral representation of languages has been debated for decades. It seems that more researchers are leaning towards the conclusion that L1 and L2 share the same classical cortical regions, although brain differences have also been found. These differences arise from multiple factors in bilinguals, such as proficiency, AoA, current L2 exposure level, etc. In a meta-analysis study, (Sebastian, et al. (2011) concluded that highly proficient bilinguals showed similar activation patterns in both languages, while low-medium-proficient bilinguals showed more widely distributed activation patterns when performing a language task in L2 compared to L1. Similar to what has been found for low proficiency bilinguals, those who acquired L2 later in life showed a greater amount of activation for L2 [for a review see Higby, et al. (2013)].

Proficiency and AoA are two important factors that are almost always highly correlated, making it difficult for researchers to separate their contributions in bilinguals. Our analysis showed that AoA had an independent contribution to bilingual brain organization in the right AG and right 
SPL while proficiency and current level of L2 exposure provided less than $10 \%$ and $1 \%$ contribution to the variance of the dependent variables, respectively. These results are consistent with the view that proficiency has a distinct role in inducing structural brain changes on top of the role played by AoA (for a review, see Li, Legault, and Litcofsky (2014) ).

The development of language-related neural organization is a dynamic process involving interactions between genetic and environmental factors (Kanai \& Rees, 2011). Whether and how changes in brain structures accompany an increase in age of second language acquisition has until now not been sufficiently investigated. The "critical period hypothesis" (CPH) received much more attention than other hypotheses. It predicts that native language proficiency cannot be obtained when learning of a second language begins after a certain age (Lenneberg, 1967). While the $\mathrm{CPH}$ is still debatable, an alternative theory, the sensorimotor hypothesis, has been developed to explain the relationship between L2 AoA and neuroplasticity (Arturo E. Hernandez \& Li, 2007). According to this hypothesis, "learning shaped the course of development in monolingual, bilingual, and nonlinguistic domains" (Arturo E. Hernandez \& Li, 2007). This model postulated that children use basic level or sensorimotor mechanisms to learn L2 while late bilinguals require additional higher-level processing. The theory received supported from several recent studies that found increased neural activities are in left/bilateral IFG for late learned words ( $\underline{\text { Fiebach, }}$ Friederici, Muller, von Cramon, \& Hernandez, 2003) and late L2 learners (A. E. Hernandez, et al., 2004; Wartenburger, et al., 2003). The angular gyrus has been implicated in the integration of basic level visual and phonological inputs (Damasio \& Damasio, 1983) and as the convergence zone for reading (Pugh, et al., 2001). The IFG, on the other hand, appeared to be critical in effortful sematic retrieval (higher-level processing). The results of the present study are consistent with sensorimotor model as we found AoA had a negative relationship with the 
volume/area of the right AG and right SPL (the earlier L2 was learned, the larger the right AG and right SPL) but a positive relationship with the measures of the right IFGor (the later L2 was learned, the larger the right IFGor). In other words, early bilinguals may be able recruit basic level linguistic information processing regions such as the right AG and right SPL while late L2 learners may have to employ higher-level processing regions like the IFG. These results implicated possible neuroanatomical substrates that reflect neuroplasticity.

In terms of neuronal plasticity of the frontal and parietal regions of the brain over development, we agree that the underdevelopment of the prefrontal cortex prior to 4 years of age may make language acquisition easier (Thompson-Schill, Ramscar, \& Chrysikou, 2009). In other words, the comparatively protracted period of PFC maturation may allow for further experiencedependent modifications as suggested by our results in the right IFGor. Even though the role of the right-hemispheric homologue of Broca's area is much less clear, the trend of brain volume and area increase in the right IFGor may reflect effortful semantic retrieval or selection processes in late bilinguals. Moreover, that none of the three variables contributed uniquely to the account of the structural differences in the right IFG indicated that several variables, such as AoA, L2 proficiency and current level of L2 exposure must be taken into consideration in resolving the puzzle of experience-dependent (Trainor, 2005) anatomical changes in the IFG. This also resonates with the idea that the frontal lobe regions are under stronger genetic control since they are in charge of some of the most important functions while the parietal lobe areas seems more responsive to environmental factors (Neubauer \& Fink, 2009).

As for exposure, Perani and her colleagues conducted the first study that indicated both AoA and the amount of language exposure may have effects on the extent of fMRI neural activation (D. Perani, et al., 2003). They proposed that decreases in left prefrontal activity might 
be a sign of decreased dependence on controlled processing and may be induced by repeated activation due to higher language exposure. Even though in our regression model, the level of L2 exposure didn't play any important role, the finding of significant negative correlation $(\mathrm{p}<0.01)$ between L2 exposure and right IFGor volume (as shown in Figure 2) is consistent with their proposal.

\subsection{What is the effect of AoA differences on regional measures?}

To a certain extent, neural organization is influenced by experiences no matter whether the experiences occur in childhood (Newman, Bavelier, Corina, Jezzard, \& Neville, 2002;

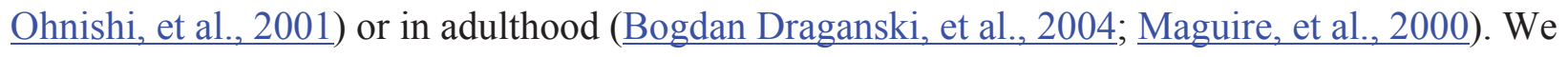
originally hypothesized that when the second language was acquired early in life, larger cortical volume in specific regions would be observed.

However, consistent with by $\underline{\mathrm{Li}}$, et al. (2014), we again found different relationships between AoA and brain regional measures: AoA had a positive relationship with the volume/area of right IFGor but a negative relationship with the volume/area of right AG and right SPL. We know that the brain follows a "back to front" rule when it matures (Gogtay, et al., 2004). That is, growth in cortical regions occurs earlier in posterior than anterior cortical regions. Peak thickness in occipital and parietal regions is attained at about 7 to 10 years of age, whereas the peak thickness in frontal and temporal regions is reached at 10 to 14 years of age (Shaw, et al., 2008). Therefore, inspired by a recent paper (Archila-Suerte, Zevin, Bunta, \& Hernandez, 2012), we propose that the reason for the different directions of relationship between ROI volume/area and AoA is that when the non-native language is acquired early in life the regions that reach maturity early in life, such as the AG and SPL in the parietal lobe, are more likely to be recruited. In contrast, if the non-native language is acquired late in life the regions that reach maturity late in 
life such as the pars orbitalis in the IFG may be recruited. In other words, brain regions may become maximally available for learning new skills at different points of time during the maturation process. This may be a way for the brain to maximize learning resources and minimize the cost in terms of energy consumption.

The changes in brain morphometry following training in prior studies are not restricted to grey matter but involve white matter as well (May, 2011). Changes in grey matter volume are in

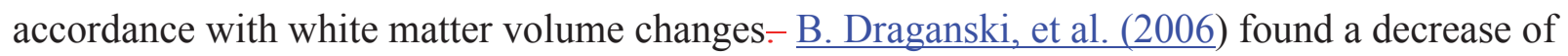
grey matter in the bilateral occipito-parietal lobe accompanied by a proportional increase of white matter in this region between scans taken 3 months before and right after participants took a sophisticated medical exam. The findings of Draganski et al. need to be viewed with caution since the VBM method they used is not very sensitive for detecting changes in white matter.

Additionally, more fine-grained methods such as microscopy are necessary to clarify the underlying cellular changes that support the macroscopic alternations in cortical volumes/area. Some possibilities are: changes in neuron size or glial cell size, and changes in synapse formation and elimination (Chklovskii, Mel, \& Svoboda, 2004; Bogdan Draganski, et al., 2004; B. Draganski \& May, 2008). Future multi-model imaging research needs to address the relationship between cellular changes and the computational capacity of cortical regions [see (Zatorre, Fields, \& Johansen-Berg, 2012)]. A detailed examination of our results in Figure 2 could reveal two important findings: one is that the relationship between AoA and the grey matter/white matter volume always has the same direction with the total volume, meaning that when there is a positive relationship between AoA and total volume of a specific region, this relationship holds for both grey matter and white matter volume. Another proposal is that reduction in grey matter volume reflects synaptic and/or neuronal pruning processes and may 
lead to more efficient processing (Kanai \& Rees, 2011). However, our results were not consistent with this hypothesis.

\subsection{VBM vs. SBM}

Previous studies suggested that VBM and SBM would produce similar results (Cerasa, et al., 2011; Lehmann, et al., 2011). Even though VBM is rapidly becoming the dominant method, it is not a replacement of ROI based analysis (Giuliani, Calhoun, Pearlson, Francis, \& Buchanan, 2005). Given that manual ROI delineation is extremely time-consuming, our semi-automatic SBM procedure could provide another choice.

We are aware that the volume differences detected by whole-brain VBM analysis would not survive stringent multiple comparison correction. However, it still provided us with useful information. It is possible that our hypothesis-driven SBM analyses reflect type I errors. Yet, with the prior FSL-VBM results at uncorrected $p=0.01$ level and in light of our hypotheses concerning the language control network, we have maximized the possibility that these results are rather from legitimate brain structure and AoA relationships. As manifested in our results, VBM and SBM might provide different type of information and should be conducted concurrently (Giuliani, et al., 2005).

\section{Conclusions}

In summary, the present results show that volumetric and area measures of the right IFGor are linked to differences in AoA, proficiency and exposure. In the left counterpart of this region, the left IFG, functional activation is known to be broader when AoA increases. The structural differences in the right IFG seen here macroscopically using MRI might reflect the recruitment of additional resources to allow processing of two languages when the non-native language is acquired relatively late in life. The total and white matter volumes of the right AG 
were reliably predicted by AoA, while AoA served as the most significant predictor for the total volume, grey matter volume and surface area of the right SPL. This finding, similarly, may reflect the need to recruit additional resources in the right parietal lobe early in life when presented with two languages. The current study suggests that the structure of the human brain is reworked by the experience of acquiring a non-native language and when considering structural changes in bilingual brains, AoA, proficiency and exposure level should all be taken into consideration.

One important limitation is that the nature of our study design did not allow us to separate effects of genetic predisposition on structural changes from structural plasticity induced by bilingual experience. It could be that inherited factors simultaneously account for individual differences in cortical volumes/thickness/area and the ability to learn a second language. Thus, further longitudinal, experimental or genetically informed studies are necessary to clarify the role of experience-dependent structural plasticity in second language acquisition (Richardson \& Price, 2009).

In terms of future directions, it is often assumed that experience-based brain plasticity is limited to functional changes instead of structural changes. Studies have illustrated that structural changes usually correspond to functional task-related activations, which corroborates a close relationship between structure and function ( $\mathrm{Li}$, et al., 2014; Richardson \& Price, 2009). We suggest that linking fMRI findings with anatomical findings might be a rich area for further research. The cellular basis underlying structural MRI findings remains to be investigated. 


\section{Acknowledgements}

This work was supported by grants from the National Science Foundation and the National Institute of Child Health and Development (HD057884). We thank Corianne Rogalsky (C.R.) and So Young Choi (S.Y. C.) for their help with brain anatomy and curve delineation. 


\section{Appendix}

\begin{tabular}{|ll|}
\hline Abbreviation & Brain Region \\
\hline ACC & Anterior cingulate cortex \\
IFG & Angular gyrus \\
IFGor & Inferior frontal gyrus \\
IFGop & Inferior frontal gyrus pars orbitalis \\
IFGpt & Inferior frontal gyrus pars opercularis \\
IPL & Inferior frontal gyrus pars triangularis \\
MFG & Inferior parietal lobule \\
PFC & Middle frontal gyrus \\
Pre-SMA & Prefrontal cortex \\
SMG & Pre-supplementary motor area \\
SPL & Supramarginal gyrus \\
STG & Superior parietal lobule \\
\hline
\end{tabular}




\section{References}

Abdul-Kareem, I. A., Stancak, A., Parkes, L. M., \& Sluming, V. (2011). Increased gray matter volume of left pars opercularis in male orchestral musicians correlate positively with years of musical performance. J Magn Reson Imaging, 33, 24-32.

Abutalebi, J. (2008). Neural aspects of second language representation and language control. Acta Psychologica, 128, 466-478.

Abutalebi, J., Della Rosa, P. A., Gonzaga, A. K., Keim, R., Costa, A., \& Perani, D. (2013). The role of the left putamen in multilingual language production. Brain Lang, 125, 307-315.

Andersson, J. L., Jenkinson, M., \& Smith, S. (2007). Non-linear registration aka Spatial normalisation FMRIB technical report TR07JA2. FMRIB Analysis Group of the University of Oxford.

Archila-Suerte, P., Zevin, J., Bunta, F., \& Hernandez, A. E. (2012). Age of acquisition and proficiency in a second language independently influence the perception of non-native speech. Bilingualism: Language and Cognition, 15, 190-201.

Badzakova-Trajkov, G., Kirk, I. J., \& Waldie, K. E. (2008). Dual-task performance in late proficient bilinguals. Laterality, 13, 201-216.

Birdsong, D. (2005). Interpreting age effects in second language acquisition. In Handbook of bilingualism: Psycholinguistic approaches (pp. 109-127). New York: Oxford University Press.

Bloch, C., Kaiser, A., Kuenzli, E., Zappatore, D., Haller, S., Franceschini, R., Luedi, G., Radue, E. W., \& Nitsch, C. (2009). The age of second language acquisition determines the variability in activation elicited by narration in three languages in Broca's and Wernicke's area. Neuropsychologia, 47, 625-633.

Buckner, R. L., Head, D., Parker, J., Fotenos, A. F., Marcus, D., Morris, J. C., \& Snyder, A. Z. (2004). A unified approach for morphometric and functional data analysis in young, old, and demented 
adults using automated atlas-based head size normalization: reliability and validation against manual measurement of total intracranial volume. Neuroimage, 23, 724-738.

Canseco-Gonzalez, E., Brehm, L., Brick, C. A., Brown-Schmidt, S., Fischer, K., \& Wagner, K. (2010). Carpet or Carcel: The effect of age of acquisition and language mode on bilingual lexical access. Language and Cognitive Processes, 25, 669-705.

Cerasa, A., Quattrone, A., Gioia, M. C., Tarantino, P., Annesi, G., Assogna, F., Caltagirone, C., De Luca, V., \& Spalletta, G. (2011). Dysbindin C-A-T haplotype is associated with thicker medial orbitofrontal cortex in healthy population. Neuroimage, 55, 508-513.

Chee, M. W., Hon, N., Lee, H. L., \& Soon, C. S. (2001). Relative language proficiency modulates BOLD signal change when bilinguals perform semantic judgments. Blood oxygen level dependent. Neuroimage, 13, 1155-1163.

Chee, M. W., Soon, C. S., Lee, H. L., \& Pallier, C. (2004). Left insula activation: a marker for language attainment in bilinguals. Proc Natl Acad Sci U S A, 101, 15265-15270.

Chee, M. W., Zheng, H., Goh, J. O., Park, D., \& Sutton, B. P. (2011). Brain structure in young and old East Asians and Westerners: comparisons of structural volume and cortical thickness. J Cogn Neurosci, 23, 1065-1079.

Chklovskii, D. B., Mel, B. W., \& Svoboda, K. (2004). Cortical rewiring and information storage. Nature, $431,782-788$.

Consonni, M., Cafiero, R., Marin, D., Tettamanti, M., Iadanza, A., Fabbro, F., \& Perani, D. (2013). Neural convergence for language comprehension and grammatical class production in highly proficient bilinguals is independent of age of acquisition. Cortex: A Journal Devoted to the Study of the Nervous System and Behavior, 49, 1252-1258.

Damasio, A. R., \& Damasio, H. (1983). The anatomic basis of pure alexia. Neurology, 33, 1573-1583.

De Bleser, R., Dupont, P., Postler, J., Bormans, G., Speelman, D., Mortelmans, L., \& Debrock, M. (2003). The organisation of the bilingual lexicon: a PET study. Journal of Neurolinguistics, 16, 439-456. 
Dehaene, S., Dupoux, E., Mehler, J., Cohen, L., Paulesu, E., Perani, D., Van de Moortele, P.-F., Lehéricy, S., \& Le Bihan, D. (1997). Anatomical variability in the cortical representation of first and second language. Neuroreport, 8, 3809-3815.

DeKeyser, R., \& Larson-Hall, J. (2005 ). What Does the Critical Period Really Mean? In Handbook of bilingualism: Psycholinguistic approaches (pp. 88-108).

Della Rosa, P. A., Videsott, G., Borsa, V. M., Canini, M., Weekes, B. S., Franceschini, R., \& Abutalebi, J. (2013). A neural interactive location for multilingual talent. Cortex, 49, 605-608.

Desikan, R. S., Segonne, F., Fischl, B., Quinn, B. T., Dickerson, B. C., Blacker, D., Buckner, R. L., Dale, A. M., Maguire, R. P., Hyman, B. T., Albert, M. S., \& Killiany, R. J. (2006). An automated labeling system for subdividing the human cerebral cortex on MRI scans into gyral based regions of interest. Neuroimage, 31, 968-980.

Dogdas, B., Shattuck, D. W., \& Leahy, R. M. (2005). Segmentation of skull and scalp in 3-D human MRI using mathematical morphology. Hum Brain Mapp, 26, 273-285.

Draganski, B., Gaser, C., Busch, V., Schuierer, G., Bogdahn, U., \& May, A. (2004). Neuroplasticity: changes in grey matter induced by training. Nature, 427, 311-312.

Draganski, B., Gaser, C., Kempermann, G., Kuhn, H. G., Winkler, J., Buchel, C., \& May, A. (2006). Temporal and spatial dynamics of brain structure changes during extensive learning. J Neurosci, 26, 6314-6317.

Draganski, B., \& May, A. (2008). Training-induced structural changes in the adult human brain. Behav Brain Res, 192, 137-142.

Duffau, H. (2006). Brain plasticity: from pathophysiological mechanisms to therapeutic applications. $J$ Clin Neurosci, 13, 885-897.

Duffau, H. (2014). The huge plastic potential of adult brain and the role of connectomics: new insights provided by serial mappings in glioma surgery. Cortex, 58, 325-337.

Elston-Guttler, K. E., Paulmann, S., \& Kotz, S. A. (2005). Who's in control? Proficiency and L1 influence on L2 processing. J Cogn Neurosci, 17, 1593-1610. 
Fiebach, C. J., Friederici, A. D., Muller, K., von Cramon, D. Y., \& Hernandez, A. E. (2003). Distinct brain representations for early and late learned words. Neuroimage, 19, 1627-1637.

Fiez, J. A. (1997). Phonology, semantics, and the role of the left inferior prefrontal cortex. Hum Brain Mapp, 5, 79-83.

Frenck-Mestre, C., Anton, J. L., Roth, M., Vaid, J., \& Viallet, F. (2005). Articulation in early and late bilinguals' two languages: evidence from functional magnetic resonance imaging. Neuroreport, $16,761-765$.

Genovese, C. R., Lazar, N. A., \& Nichols, T. (2002). Thresholding of statistical maps in functional neuroimaging using the false discovery rate. Neuroimage, 15, 870-878.

Giuliani, N. R., Calhoun, V. D., Pearlson, G. D., Francis, A., \& Buchanan, R. W. (2005). Voxel-based morphometry versus region of interest: a comparison of two methods for analyzing gray matter differences in schizophrenia. Schizophr Res, 74, 135-147.

Gogtay, N., Giedd, J. N., Lusk, L., Hayashi, K. M., Greenstein, D., Vaituzis, A. C., Nugent, T. F., 3rd, Herman, D. H., Clasen, L. S., Toga, A. W., Rapoport, J. L., \& Thompson, P. M. (2004). Dynamic mapping of human cortical development during childhood through early adulthood. Proc Natl Acad Sci U S A, 101, 8174-8179.

Good, C. D., Johnsrude, I. S., Ashburner, J., Henson, R. N., Friston, K. J., \& Frackowiak, R. S. (2001). A voxel-based morphometric study of ageing in 465 normal adult human brains. Neuroimage, 14, 21-36.

Green, D. W., \& Abutalebi, J. (2013). Language control in bilinguals: The adaptive control hypothesis. Journal of Cognitive Psychology, 1-16.

Grogan, A., Parker, J., Ali, N., Crinion, J., Orabona, S., Mechias, M. L., Ramsden, S., Green, D. W., \& Price, C. J. (2012). Structural correlates for lexical efficiency and number of languages in nonnative speakers of English. Neuropsychologia, 50, 1347-1352. 
Gurd, J. M., Amunts, K., Weiss, P. H., Zafiris, O., Zilles, K., Marshall, J. C., \& Fink, G. R. (2002).

Posterior parietal cortex is implicated in continuous switching between verbal fluency tasks: an fMRI study with clinical implications. Brain, 125, 1024-1038.

Hernandez, A. E. (2009). Language switching in the bilingual brain: What's next? Brain Lang, 109, 133140.

Hernandez, A. E., Hofmann, J., \& Kotz, S. A. (2007). Age of acquisition modulates neural activity for both regular and irregular syntactic functions. Neuroimage, 36, 912-923.

Hernandez, A. E., Kotz, S. A., Hofmann, J., Valentin, V. V., Dapretto, M., \& Bookheimer, S. Y. (2004). The neural correlates of grammatical gender decisions in Spanish. Neuroreport, 15, 863-866.

Hernandez, A. E., \& Li, P. (2007). Age of Acquisition: Its Neural and Computational Mechanisms. Psychological Bulletin, 133, 638-650.

Higby, E., Kim, J., \& Obler, L. K. (2013). Multilingualism and the Brain. Annual Review of Applied Linguistics, 33, 68-101.

Hull, R., \& Vaid, J. (2007). Bilingual language lateralization: A meta-analytic tale of two hemispheres. Neuropsychologia, 45, 1987-2008.

Indefrey, P. (2006). A Meta-analysis of Hemodynamic Studies on First and Second Language Processing: Which Suggested Differences Can We Trust and What Do They Mean? Language Learning, 56, 279-304.

Isel, F., Baumgaertner, A., Thrän, J., Meisel, J. M., \& Büchel, C. (2010). Neural circuitry of the bilingual mental lexicon: Effect of age of second language acquisition. Brain Cogn, 72, 169-180.

Jeong, H., Sugiura, M., Sassa, Y., Haji, T., Usui, N., Taira, M., Horie, K., Sato, S., \& Kawashima, R. (2007). Effect of syntactic similarity on cortical activation during second language processing: a comparison of English and Japanese among native Korean trilinguals. Hum Brain Mapp, 28, 194204.

Jia, G., Aaronson, D., \& Wu, Y. (2002). Long-term language attainment of bilingual immigrants: Predictive variables and language group differences. Applied Psycholinguistics, 23, 599-621. 
Joshi, A., Shattuck, D., Pantazis, D., Li, Q. Z., Damasio, H., Leahy, R., \& Ieee. (2009). Optimization of Landmark Selection for Cortical Surface Registration. In Cvpr: 2009 Ieee Conference on Computer Vision and Pattern Recognition, Vols 1-4 (pp. 699-706). New York: Ieee.

Joshi, A., Shattuck, D., Thompson, P., \& Leahy, R. (2007). Brain image registration using cortically constrained harmonic mappings. Inf Process Med Imaging, 20, 359-371.

Joshi, A. A., Pantazis, D., Li, Q., Damasio, H., Shattuck, D. W., Toga, A. W., \& Leahy, R. M. (2010). Sulcal set optimization for cortical surface registration. Neuroimage, 50, 950-959.

Joshi, A. A., Shattuck, D. W., \& Leahy, R. M. (2012). A Fast and Accurate Method for Automated Cortical Surface Registration and Labeling. In Proc. WBIR LNCS Springer (pp. 180-189).

Kanai, R., \& Rees, G. (2011). The structural basis of inter-individual differences in human behaviour and cognition. Nat Rev Neurosci, 12, 231-242.

Kim, J.-H., Lee, J.-M., Jo, H. J., Kim, S. H., Lee, J. H., Kim, S. T., Seo, S. W., Cox, R. W., Na, D. L., Kim, S. I., \& Saad, Z. S. (2010). Defining functional SMA and pre-SMA subregions in human MFC using resting state fMRI: Functional connectivity-based parcellation method. Neuroimage, 49, 2375-2386.

Kim, K. H., Relkin, N. R., Lee, K. M., \& Hirsch, J. (1997). Distinct cortical areas associated with native and second languages. Nature, 388, 171-174.

Klein, D., Mok, K., Chen, J. K., \& Watkins, K. E. (2013). Age of language learning shapes brain structure: A cortical thickness study of bilingual and monolingual individuals. Brain Lang.

Klein, D., Zatorre, R. J., Chen, J. K., Milner, B., Crane, J., Belin, P., \& Bouffard, M. (2006). Bilingual brain organization: a functional magnetic resonance adaptation study. Neuroimage, 31, 366-375.

Kovelman, I., Baker, S. A., \& Petitto, L.-A. (2008). Age of first bilingual language exposure as a new window into bilingual reading development. Bilingualism: Language and Cognition, 11, 203-223.

Le, T. H., Pardo, J. V., \& Hu, X. (1998). 4 T-fMRI study of nonspatial shifting of selective attention: cerebellar and parietal contributions. J Neurophysiol, 79, 1535-1548. 
Lehmann, M., Crutch, S. J., Ridgway, G. R., Ridha, B. H., Barnes, J., Warrington, E. K., Rossor, M. N., \& Fox, N. C. (2011). Cortical thickness and voxel-based morphometry in posterior cortical atrophy and typical Alzheimer's disease. Neurobiol Aging, 32, 1466-1476.

Lenneberg, E. H. (1967). Biological foundations of language. New York: Wiley, (Chapter).

Li, P., Legault, J., \& Litcofsky, K. A. (2014). Neuroplasticity as a function of second language learning: Anatomical changes in the human brain. Cortex, 58, 301-324.

Maguire, E. A., Gadian, D. G., Johnsrude, I. S., Good, C. D., Ashburner, J., Frackowiak, R. S., \& Frith, C. D. (2000). Navigation-related structural change in the hippocampi of taxi drivers. Proceedings of the National Academy of Sciences, 97, 4398-4403.

Mahendra, N., Plante, E., Magloire, J., Milman, L., \& Trouard, T. P. (2003). FMRI variability and the localization of languages in the bilingual brain. Neuroreport, 14, 1225-1228.

Mårtensson, J., Eriksson, J., Bodammer, N. C., Lindgren, M., Johansson, M., Nyberg, L., \& Lövdén, M. (2012). Growth of language-related brain areas after foreign language learning. Neuroimage, 63, 240.

May, A. (2011). Experience-dependent structural plasticity in the adult human brain. Trends Cogn Sci, 15, $475-482$.

Mayberry, R. I., Chen, J. K., Witcher, P., \& Klein, D. (2011). Age of acquisition effects on the functional organization of language in the adult brain. Brain Lang, 119, 16-29.

Mechelli, A., Crinion, J., Noppeney, U., Doherty, J., Ashburner, J., Frackowiak, R. S., \& Price, C. J. (2004). Structural plasticity in the bilingual brain- Proficiency in a second language and age at acquisition affect grey-matter density. Nature, 431 .

Musso, M., Moro, A., Glauche, V., Rijntjes, M., Reichenbach, J., Buchel, C., \& Weiller, C. (2003). Broca's area and the language instinct. Nat Neurosci, 6, 774-781.

Nee, D. E., Wager, T. D., \& Jonides, J. (2007). Interference resolution: insights from a meta-analysis of neuroimaging tasks. Cogn Affect Behav Neurosci, 7, 1-17. 
Neubauer, A. C., \& Fink, A. (2009). Intelligence and neural efficiency. Neuroscience \& Biobehavioral Reviews, 33, 1004-1023.

Newman, A. J., Bavelier, D., Corina, D., Jezzard, P., \& Neville, H. J. (2002). A critical period for right hemisphere recruitment in American Sign Language processing. Nat Neurosci, 5, 76-80.

Ohnishi, T., Matsuda, H., Asada, T., Aruga, M., Hirakata, M., Nishikawa, M., Katoh, A., \& Imabayashi, E. (2001). Functional anatomy of musical perception in musicians. Cerebral cortex (New York, N.Y. : 1991), 11, 754-760.

Oldfield, R. C. (1971). The assessment and analysis of handedness: the Edinburgh inventory. Neuropsychologia, 9, 97-113.

Pantazis, D., Joshi, A., Jiang, J., Shattuck, D. W., Bernstein, L. E., Damasio, H., \& Leahy, R. M. (2010). Comparison of landmark-based and automatic methods for cortical surface registration. Neuroimage, 49, 2479-2493.

Park, H. R., Badzakova-Trajkov, G., \& Waldie, K. E. (2012). Language lateralisation in late proficient bilinguals: a lexical decision fMRI study. Neuropsychologia, 50, 688-695.

Peng, G., \& Wang, W. S. Y. (2011). Hemisphere lateralization is influenced by bilingual status and composition of words. Neuropsychologia, 49, 1981-1986.

Perani, D., \& Abutalebi, J. (2005). The neural basis of first and second language processing. Current Opinion in Neurobiology, 15, 202-206.

Perani, D., Abutalebi, J., Paulesu, E., Brambati, S., Scifo, P., Cappa, S. F., \& Fazio, F. (2003). The role of age of acquisition and language usage in early, high-proficient bilinguals: An fMRI study during verbal fluency. Hum Brain Mapp, 19, 170-182.

Perani, D., Paulesu, E., Galles, N. S., Dupoux, E., Dehaene, S., Bettinardi, V., Cappa, S. F., Fazio, F., \& Mehler, J. (1998). The bilingual brain - Proficiency and age of acquisition of the second language. Brain, 121, 1841-1852.

Poldrack, R. A. (2007). Region of interest analysis for fMRI. Soc Cogn Affect Neurosci, 2, 67-70. 
Pugh, K. R., Mencl, W. E., Jenner, A. R., Katz, L., Frost, S. J., Lee, J. R., Shaywitz, S. E., \& Shaywitz, B. A. (2001). Neurobiological studies of reading and reading disability. J Commun Disord, 34, 479492.

Ressel, V., Pallier, C., Ventura-Campos, N., Díaz, B., Roessler, A., Ávila, C., \& Sebastián-Gallés, N. (2012). An effect of bilingualism on the auditory cortex. The Journal of neuroscience, 32, 1659716601.

Richardson, F. M., \& Price, C. J. (2009). Structural MRI studies of language function in the undamaged brain. Brain Struct Funct, 213, 511-523.

Sebastian, R., Laird, A. R., \& Kiran, S. (2011). Meta-analysis of the neural representation of first language and second language. Applied Psycholinguistics, 32, 799-819.

Seghier, M. L. (2013). The angular gyrus: multiple functions and multiple subdivisions. Neuroscientist, $19,43-61$.

Shattuck, D. W., Joshi, A. A., Pantazis, D., Kan, E., Dutton, R. A., Sowell, E. R., Thompson, P. M., Toga, A. W., \& Leahy, R. M. (2009). Semi-automated method for delineation of landmarks on models of the cerebral cortex. $J$ Neurosci Methods, 178, 385-392.

Shattuck, D. W., \& Leahy, R. M. (2002). BrainSuite: an automated cortical surface identification tool. Med Image Anal, 6, 129-142.

Shattuck, D. W., Sandor-Leahy, S. R., Schaper, K. A., Rottenberg, D. A., \& Leahy, R. M. (2001). Magnetic resonance image tissue classification using a partial volume model. Neuroimage, 13, 856-876.

Shaw, P., Kabani, N. J., Lerch, J. P., Eckstrand, K., Lenroot, R., Gogtay, N., Greenstein, D., Clasen, L., Evans, A., Rapoport, J. L., Giedd, J. N., \& Wise, S. P. (2008). Neurodevelopmental trajectories of the human cerebral cortex. J Neurosci, 28, 3586-3594.

Storey, J. D. (2002). A direct approach to false discovery rates. Journal of the Royal Statistical Society: Series B (Statistical Methodology), 64, 479-498. 
Thompson-Schill, S. L., Ramscar, M., \& Chrysikou, E. G. (2009). Cognition without control: When a little frontal lobe goes a long way. Curr Dir Psychol Sci, 18, 259-263.

Trainor, L. J. (2005). Are there critical periods for musical development? Dev Psychobiol, 46, 262-278.

Vaid, J. (1983). Bilingualism and brain lateralization. In S. Segalowitz (Ed.), Language function with brain organization (pp. 315-339). New York: Academic Press.

Vingerhoets, G., Van Borsel, J., Tesink, C., van den Noort, M., Deblaere, K., Seurinck, R., Vandemaele, P., \& Achten, E. (2003). Multilingualism: an fMRI study. Neuroimage, 20, 2181-2196.

Waldron, E. J., \& Hernandez, A. E. (2013). The role of age of acquisition on past tense generation in Spanish-English bilinguals: an fMRI study. Brain Lang, 125, 28-37.

Wang, Y., Xiang, J., Vannest, J., Holroyd, T., Narmoneva, D., Horn, P., Liu, Y., Rose, D., DeGrauw, T., \& Holland, S. (2011). Neuromagnetic measures of word processing in bilinguals and monolinguals. Clinical Neurophysiology, 122, 1706-1717.

Wartenburger, I., Heekeren, H. R., Abutalebi, J., Cappa, S. F., Villringer, A., \& Perani, D. (2003). Early setting of grammatical processing in the bilingual brain. Neuron, 37, 159-170.

Wattendorf, E., \& Festman, J. (2008). Images of the multilingual brain: the effect of age of second language acquisition. Annual Review of Applied Linguistics, 28, 3-24.

Weber-Fox, C. M., \& Neville, H. J. (1996). Maturational constraints on functional specializations for language processing: ERP and behavioral evidence in bilingual speakers. $J$ Cogn Neurosci, 8, 231-256.

Wible, C. G., Shenton, M. E., Hokama, H., \& et al. (1995). Prefrontal cortex and schizophrenia: A quantitative magnetic resonance imaging study. Arch Gen Psychiatry, 52, 279-288.

Woods, R. P., Grafton, S. T., Holmes, C. J., Cherry, S. R., \& Mazziotta, J. C. (1998). Automated image registration: I. General methods and intrasubject, intramodality validation. J Comput Assist Tomogr, 22, 139-152.

Woollett, K., \& Maguire, E. A. (2011). Acquiring "the Knowledge" of London's layout drives structural brain changes. Curr Biol, 21, 2109-2114. 
Yoon, U., Fahim, C., Perusse, D., \& Evans, A. C. (2010). Lateralized genetic and environmental influences on human brain morphology of 8-year-old twins. Neuroimage, 53, 1117-1125.

Zatorre, R. J., Fields, R. D., \& Johansen-Berg, H. (2012). Plasticity in gray and white: neuroimaging changes in brain structure during learning. Nature neuroscience, 15, 528-536.

Zou, L., Ding, G., Abutalebi, J., Shu, H., \& Peng, D. (2012). Structural plasticity of the left caudate in bimodal bilinguals. Cortex, 48, 1197-1206. 
Table 1. Summary of descriptive statistics in self-reported language history questionnaire.

\begin{tabular}{ccccccc}
\hline & \multicolumn{3}{l}{ Native language (English) } & \multicolumn{3}{l}{ Non-native language } \\
\cline { 2 - 7 } Language history measures & Mean & SD & Range & Mean & SD & Range \\
\hline Age of acquisition (years) & 0.97 & 1.58 & $0.00-6.00$ & 9.47 & 7.19 & $0.00-21.00$ \\
Self-reported proficiency & & & & & & \\
Reading & 6.89 & 0.40 & $5.00-7.00$ & 3.28 & 1.54 & $1.00-6.00$ \\
Writing & 6.78 & 0.59 & $4.00-7.00$ & 2.97 & 1.42 & $1.00-6.00$ \\
Listening & 6.92 & 0.37 & $5.00-7.00$ & 3.81 & 1.79 & $1.00-7.00$ \\
Speaking & 6.83 & 0.51 & $5.00-7.00$ & 3.72 & 1.63 & $1.00-7.00$ \\
Current level of L2 exposure (\% of day) & 91.33 & 10.49 & $40-100$ & 10.16 & 12.47 & $0-60$ \\
\hline
\end{tabular}


Table 2. Two sets of parameters used in N4 bias field correction procedure

\begin{tabular}{|c|c|c|c|c|c|}
\hline & Number of iterations & Convergence threshold & BSpline grid resolution & Spline distance & Shrink factor \\
\hline Set 1 & $150,140,130$ & 0.0001 & $1,1,1$ & 0.00 & 4 \\
\hline Set 2 & $150,140,130$ & 0.0001 & $2,2,2$ & 0.00 & 2 \\
\hline
\end{tabular}


Table 3. Segmented brain regions

\section{Cortex}

Frontal lobe

Parietal lobe

Frontal-parietal junction

Temporal lobe
Superior frontal gyrus

Middle frontal gyrus

Pars opercularis

Pars triangularis

Pars orbitalis

Precentral gyrus

Prefrontal cortex

Pre-Supplementary motor area

Transverse frontal gyrus

Gyrus rectus

Anterior orbito-frontal gyrus

Middle orbito-frontal gyrus

Lateral orbito-frontal gyrus

Posterior orbito-frontal gyrus

Postcentral gyrus

Supramarginal gyrus

Angular gyrus

Superior parietal gyrus

Precuneus

Paracentral lobule

Temporal pole

Superior temporal gyrus

Transverse temporal gyrus

Middle temporal gyrus

Inferior temporal gyrus

Fusiform gyrus
Occipital lobe

Superior occipital gyrus

Middle occipital gyrus

Inferior occipital gyrus

Lingual gyrus

Cuneus

Limbic lobe

Subcortex

Insular lobe
Insula
Hippocampus

Parahippocampal Gyrus

Cingulate gyrus

Anterior cingulate cortex

Subcallosal area
Thalamus

Amygdala

Caudate nucleus

Putamen

Globus pallidus

Nucleus accumbens

Claustrum

Basal forebrain

Lateral geniculate nucleus

Medial geniculate nucleus

Superior colliculus

Inferior colliculus

Mamillary body 
Table 4. Multiple regressions of grey matter volume, white matter volume, total volume and cortical area in FDR correction survived regions.

\begin{tabular}{|c|c|c|c|c|c|c|c|c|c|}
\hline \multicolumn{2}{|c|}{ Outcome variable } & \multirow{2}{*}{$\begin{array}{l}\text { Predictor variable } \\
\text { AoA }\end{array}$} & \multirow{2}{*}{$\begin{array}{l}\mathrm{R}^{2} \\
0.302\end{array}$} & \multirow{2}{*}{$\begin{array}{l}\mathrm{F} \\
4.610\end{array}$} & \multirow{2}{*}{\begin{tabular}{|l} 
Sig. model \\
$0.009 * *$
\end{tabular}} & \multirow[t]{2}{*}{$\mathrm{b}$} & \multirow[t]{2}{*}{$\mathrm{t}$} & \multirow{2}{*}{$\begin{array}{l}\text { Sig. predictor } \\
0.229\end{array}$} & \multirow{2}{*}{$\begin{array}{l}\text { Variance predicted } \\
\text { by AoA }\end{array}$} \\
\hline Total & R.IFGor & & & & & & & & \\
\hline \multirow[t]{8}{*}{ volume } & & Proficiency & & & & & & 0.865 & \\
\hline & & Exposure & & & & & & 0.059 & \\
\hline & R.AG & AoA & 0.254 & 3.639 & $0.023 *$ & -230.971 & -2.654 & $0.012 *$ & $16.403 \%$ \\
\hline & & Proficiency & & & & & & 0.092 & \\
\hline & & Exposure & & & & & & 0.970 & \\
\hline & R.SPL & AoA & 0.333 & 5.337 & $0.004 * *$ & -178.670 & -3.008 & $0.005^{* *}$ & $18.836 \%$ \\
\hline & & Proficiency & & & & & & 0.233 & \\
\hline & & Exposure & & & & & & 0.583 & \\
\hline Grey & R.IFGor & AoA & 0.299 & 4.545 & $0.009 * *$ & & & 0.281 & \\
\hline matter & & Proficiency & & & & & & 0.958 & \\
\hline \multirow[t]{4}{*}{ volume } & & Exposure & & & & & & 0.053 & \\
\hline & R.SPL & AoA & 0.352 & 5.792 & $0.003 * *$ & -120.590 & -3.097 & $0.004 * *$ & $19.448 \%$ \\
\hline & & Proficiency & & & & & & 0.188 & \\
\hline & & Exposure & & & & & & 0.563 & \\
\hline White & R.IFGor & AoA & 0.236 & 3.292 & $0.033^{*}$ & & & 0.171 & \\
\hline matter & & Proficiency & & & & & & 0.873 & \\
\hline \multirow[t]{4}{*}{ volume } & & Exposure & & & & & & 0.192 & \\
\hline & R.AG & AoA & 0.315 & 4.900 & $0.006^{* *}$ & -93.862 & -3.148 & $0.004 * *$ & $21.252 \%$ \\
\hline & & Proficiency & & & & & & 0.122 & \\
\hline & & Exposure & & & & & & 0.992 & \\
\hline Cortical & R.IFGor & AoA & 0.308 & 4.162 & $0.015^{*}$ & & & 0.338 & \\
\hline \multirow[t]{5}{*}{ area } & & Proficiency & & & & & & 0.529 & \\
\hline & & Exposure & & & & & & 0.127 & \\
\hline & R.SPL & AoA & 0.270 & 3.453 & $0.030 *$ & -45.273 & -2.632 & $0.014 *$ & $18.063 \%$ \\
\hline & & Proficiency & & & & & & 0.661 & \\
\hline & & Exposure & & & & & & 0.601 & \\
\hline
\end{tabular}

Note. AoA, proficiency and exposure were added in the model as predictors. When none of the three predictors had a statistically significant contribution, we reported each predictor's $p$ value in the model. When any of the three predictors was a significant predictor, we reported the $t, b, p$ values and variance explained by this predictor $\left(p<0.05^{*}, p<0.01 * *\right) . \mathrm{R}=$ right; IFGor= inferior frontal gyrus pars orbitalsi; $\mathrm{AG}=$ angular gyrus; $\mathrm{SPL}=$ Superior parietal lobule. 